\title{
Actualizaciones en la mordedura de perro
}

\author{
Updates on the dog bite \\ Mauricio De la Concha Tiznado, * Francisco José Flores Palomar,* Rodolfo Iván Lara Ruiz, * \\ Arantxa Cabadas Contreras, ${ }^{\ddagger}$ José Andrés García Huitrón ${ }^{\S}$
}

\section{Resumen}

La mordedura de perro es un padecimiento común en nuestro medio. La mayoría de los casos son atendidos por el Servicio de Urgencias, y de éstos sólo un porcentaje de pacientes que presentan complicaciones amerita hospitalización. Se debe realizar una adecuada exploración debido a que el tipo de herida y los hallazgos clínicos modifican nuestra conducta terapéutica. Los exámenes de laboratorio y gabinete nos ayudan a confirmar los casos en los que se sospechen lesiones asociadas y complicaciones. Actualmente, se encuentran en discusión las indicaciones y contraindicaciones para el cierre de la herida. El tratamiento antibiótico profiláctico no disminuye la incidencia de complicaciones infecciosas y los esquemas terapéuticos sólo deben de iniciarse una vez confirmada la infección.

Palabras clave: Mordedura de perro, manejo, actualización, herida, infección.

\section{INTRODUCCIÓN}

Una mordedura es una herida o punción hecha por los dientes o cualquier parte de la boca de un organismo vivo. Las mordeduras de animales y humanos representan un gran problema de salud en México, pues cursan con una alta morbilidad $y$, en casos aislados, con mortalidad. ${ }^{1}$

\footnotetext{
* Residente de Cirugía General. Facultad Mexicana de Medicina Universidad La Salle.

* Interno de Pregrado. Escuela de Medicina, Universidad Anáhuac México Norte.

§ Interno de Pregrado. Escuela de Medicina, Universidad Anáhuac Querétaro.
}

Hospital Ángeles Pedregal. Ciudad de México, México.

Correspondencia:

Mauricio De la Concha Tiznado

Correo electrónico: drmauriciodelaconcha@gmail.com

Aceptado: 10-03-2020.

www.medigraphic.com/actamedica

\section{Abstract}

Dog bites is a common condition in our environment. Most cases are treated in the emergency department and only a percentage of patients who have complications merit hospitalization. An adequate examination should be performed because the type of wound and the clinical findings modify the therapeutic behavior. Laboratory and imaging tests help us confirm cases in which associated injuries and complications are suspected. The indications and contraindications for wound closure are currently under discussion. Prophylactic antibiotic treatment does not reduce the incidence of infectious complications and therapeutic regimens should only be initiated once an infection is confirmed.

Keywords: Dog bite, management, update, wound, infection.

Existe una alta incidencia en el país, alrededor de 110.44 por cada 100 mil habitantes. Los estados más afectados son Durango, Hidalgo, Puebla, Ciudad de México, Estado de México y San Luis Potosí. El año pasado, la Dirección General de Epidemiología registró que el Estado de México y la Ciudad de México fueron los estados con más casos por mordeduras de perro, alcanzando 17,172 y 14,441 casos, respectivamente. ${ }^{2}$

El objetivo del presente artículo es realizar una revisión en PubMed de la literatura sobre la mordedura, dar a conocer la epidemiología y factores de riesgo, correlacionar los hallazgos clínicos con las probables complicaciones y exponer las principales alternativas de tratamiento.

\section{EPIDEMIOLOGÍA Y FACTORES DE RIESGO}

En Estados Unidos, las mordeduras de perro representan $1 \%$ de las visitas de trauma en los Servicios de Urgencias; ${ }^{3-5}$ de éstas, $50 \%$ de las mordeduras de perros y gatos requiere atención médica y $2.5 \%$ requiere hospitalización. $13,4,6$

Las mordeduras por perros constituyen de 60 a $80 \%$ y la de los gatos causa de 20 a 30\%., ${ }^{78}$ Las localizaciones más frecuentes 
son mano (articulación metacarpofalángica y falanges proximales del segundo al quinto dedo) de 18 a 36\%, en el brazo de 17 a $20 \%$, en la pierna de 5 a $15 \%$ y en la cara de 4 a $11 \%$. ${ }^{7,9}$

El mayor porcentaje por mordeduras lo constituyen niños (20-38\% de las víctimas), de los cuales 25\% son menores de seis años y $34 \%$ tiene entre seis y 17 años. ${ }^{4,7,8} \mathrm{En}$ niños menores de nueve años, los sitios más frecuentemente afectados son cabeza y cuello, aunque en mayores de 10 años, los brazos y piernas. ${ }^{6,8,10}$ Los hombres generalmente se ven afectados por perros y las mujeres por gatos. ${ }^{7,9}$

De 10 a $20 \%$ de las heridas por mordedura se complicarán con infección. ${ }^{1,7}$ La infección es más probable cuando las heridas son profundas o si se aloja un cuerpo extraño. ${ }^{1}$ Asimismo, las mordeduras de gato tienen una mayor incidencia de infección en comparación con otros mamíferos, presentándose en 30 a 50\%, 3,6,7,10 seguidas por las de humanos con un porcentaje que va de 15 a $25 \%$, y finalmente las de perros con un 5 a $25 \% .^{7}$

\section{CLÍNICA}

Se deben clasificar las heridas según el patrón que presenten, pudiendo ser laceración, heridas punzantes, abrasión, avulsión, aplastamiento y amputación. ${ }^{3}$ Es importante documentar en cada una: la localización, el ancho, lo largo, la profundidad y la presencia de cuerpos extraños. ${ }^{3,7} \mathrm{Se}$ debe evaluar el área distal a la lesión buscando la integridad neurovascular. La extensión y flexión en articulaciones puede revelar defectos pequeños o parciales en tendones, y fracturas $^{3}$ (Tabla 1).

Aproximadamente, $60 \%$ de las heridas por perro son punzantes, $10 \%$ son laceraciones y un $30 \%$ son combinaciones de varias. ${ }^{5,11,12}$ Debido a que los dientes de perro son más amplios y romos, generalmente sólo causan laceraciones que involucran tejidos superficiales, lo cual podría explicar por qué sólo $25 \%$ de los casos se complican con infecciones. ${ }^{6}$

Se pueden observar datos de infección como fiebre, edema, eritema, drenaje purulento de la herida, celulitis, abscesos y linfadenopatía. ${ }^{3,7}$ La mayoría de las infecciones en la herida son purulentas sin la formación de absceso (58\%), seguida de infecciones no purulentas con celulitis o linfangitis (30\%) y con formación de abscesos (12\%). ${ }^{13}$ La

Tabla 1: Clasificación de heridas de Rueff.

\begin{tabular}{cl} 
Grado & Características \\
\hline I & Superficiales \\
II & Se extiende de la piel, fascia, músculo o cartílago \\
III & Necrosis y pérdida de tejido
\end{tabular}

\begin{tabular}{|c|c|}
\hline Aerobios & Anaerobios \\
\hline $\begin{array}{l}\text { Pasteurella spp. } \\
\text { Pasteurella multocida } \\
\text { Pasteurella canis } \\
\text { Streptococcus spp. } \\
\text { Staphylococcus spp. } \\
\text { Neisseria spp. } \\
\text { Capnocytophaga canimorsus } \\
\text { Moraxella spp. }\end{array}$ & $\begin{array}{l}\text { Fusobacterium spp. } \\
\text { Bacteroides spp. } \\
\text { Porphyromonas spp. } \\
\text { Prevotella spp. } \\
\text { Propionibacterium } \\
\text { Preptostreptococcus }\end{array}$ \\
\hline
\end{tabular}

celulitis causada por Staphylococcus y Streptococcus es difusa y menos severa que la causada por Pasteurella multocida.

Los signos de infección no se presentan con la misma latencia, por ejemplo, las infecciones por P. multocida presentan manifestaciones en las primeras 12 a 24 horas, ${ }^{1,4,5,8,9}$ comparado con Capnocytophaga canimorsus, la cual puede llegar a presentar datos clínicos después de los cinco a ocho días. ${ }^{7}$

\section{COMPLICACIONES INFECCIOSAS}

La complicación más frecuente es la infección de la herida. De 30 a $60 \%$ presenta una combinación entre patógenos aerobios y anaerobios (Tabla 2); 6,7,11 en promedio, heridas provocadas por perros y gatos contienen entre dos a cinco especies diferentes de bacterias. ${ }^{6,9}$ El número de especies de patógenos va a depender del tipo de lesión que se presente; se ha reportado una mediana de 7.5 microorganismos en abscesos, cinco en heridas purulentas y dos en heridas no purulentas. ${ }^{7}$

Los agentes anaerobios están relacionados con abscesos, estafilococos y estreptococos, si bien predominan en heridas no abscedadas. ${ }^{1}$ Los patógenos más comunes presentes en las lesiones son Pasteurella spp., Staphylococcus spp. (incluidos MRSA) y Streptococcus spp. ${ }^{1,7}$ Entre los miembros de Pasteurella spp., Pasteurella canis se posiciona en el primer lugar de las infecciones por mordedura de perro, siendo ésta la más frecuentemente aislada. ${ }^{11}$

Otro microorganismo que ha tomado relevancia en los últimos años es un bacilo Gram negativo: Capnocytophaga, que se ha aislado en $24 \%$ de las mordeduras de perro y $17 \%$ de las mordeduras de gatos. Éste se asocia con septicemia, falla renal y necrosis de las extremidades. ${ }^{4,14}$

\section{DIAGNÓSTICO}

Se deberá obtener información detallada sobre el incidente, tal como tiempo, lugar y circunstancia, especie del animal y estado de salud actual de éste. ${ }^{3,7}$ La exploración física 
revela datos de lesiones cutáneas, hematomas, infecciones subcutáneas y fracturas. ${ }^{3}$

Es importante cuestionar al paciente sobre alergias, inmunizaciones, presencia de implantes (válvulas cardiacas, prótesis articulares), tratamientos previos y condiciones crónicas, como inmunodeficiencias. ${ }^{3}$

La tinción de Gram y los cultivos para aerobios y anaerobios están indicados en caso de sospecha de infección local y en aquellos pacientes que se presenten ocho horas posteriores al evento. El seguimiento se realizará por un mínimo de siete a 10 días. ${ }^{3,7}$ Cuando se presenten datos de infección sistémica, la proteína $\mathrm{C}$ reactiva y la sedimentación globular pueden ser de ayuda. ${ }^{3}$

Deben considerarse los exámenes de laboratorio y gabinete en pacientes con riesgo de complicaciones. Por ello, obtener radiografía, tomografía computarizada o resonancia magnética puede demostrar la presencia de cuerpos extraños, lesiones articulares, tendones y fracturas que no sean evidentes en la clínica; el ultrasonido aporta información importante en caso de sospecha de lesión vascular o de abscesos. ${ }^{3,7}$

\section{TRATAMIENTO}

Se recomienda el uso analgésico para realizar una adecuada exploración de la lesión y manejo del dolor posterior al tratamiento. El manejo del dolor se puede realizar con lidocaína o tetracaína tópica; en niños, otra opción es posible utilizar midazolam como sedación y analgesia. ${ }^{1}$

\section{Manejo de la herida \\ Limpieza de la herida}

Se practica incisión y drenaje de heridas fluctuantes o abscedadas. Se debe consultar con el cirujano en caso de abscesos que están sobre zonas de compromiso funcional o de riesgo alto de cicatrices desfigurantes. ${ }^{1}$

$$
\text { Irrigación }
$$

Se debe irrigar abundantemente la herida a razón de 100 a $200 \mathrm{~mL}$ por pulgada con cloruro de sodio a $0.9 \%$, povidona yodada a $1 \%$ o cloruro de benzalconio a $1 \%$. Un catéter de jeringa de $20 \mathrm{~mL}$ o mayor proporciona la presión suficiente; asimismo, se debe de evitar la irrigación con presión alta para no dañar los tejidos y no inocular profundamente bacterias o cuerpos extraños. 1,3,4,8,15,16

\section{Debridación y retiro de cuerpos extraños}

La debridación quirúrgica del tejido desvitalizado es sumamente eficaz, pero está limitada por el sitio anatómico.
Ésta realiza mediante la escisión de los bordes irregulares y dentados, y de restos de tejidos no viables que aumenten la posibilidad de inoculación, replicación bacteriana y posterior necrosis. $1,7,17$

En heridas en cabeza y cara, sin embargo, no puede ser extensa, como en las extremidades en donde existen más posibilidades para la reconstrucción del tejido. En articulaciones infectadas, una segunda revisión 48 horas después previene la destrucción y la inmovilidad. Se recomienda la inmovilización y fisioterapia temprana. ${ }^{7}$

\section{Cierre}

La sutura de la herida es un tema en discusión debido al riesgo de infección. Aunque todavía no existe un consenso, en general, el manejo temprano de las heridas parece estar correlacionado con rangos menores de infección y con mejores resultados estéticos. ${ }^{15}$

Tradicionalmente, se sugiere manejar las heridas con cierre secundario para disminuir el riesgo de infección, pero actualmente existen series en las que se observa que el cierre primario no necesariamente aumenta su incidencia. $4,7,15,18-20$

Los autores más conservadores retrasan el cierre de mordeduras si se encuentran infectadas, mientras que otros recomiendan una adecuada irrigación y debridación antes del cierre, sin encontrar diferencia entre el cierre primario y secundario. 4,8,15,19-21

Actualmente, existen propuestas para el cierre primario en heridas faciales, grandes, desfigurantes o con menos de ocho a 12 horas de evolución. ${ }^{3,4,7,8}$ Las heridas en cara deben suturarse pronto por las infrecuentes complicaciones infecciosas; además, se ha tenido un buen resultado estético, gracias al excelente riego sanguíneo de la cara y cuero cabelludo. ${ }^{1,22}$

Las contraindicaciones para el cierre son heridas faciales y en extremidades de más de 12 a 24 horas de evolución, mordeduras punzantes en mano o en sitios con implantes, mordeduras asociadas con lesiones por aplastamiento y en pacientes inmunocomprometidos. ${ }^{1,3,4,7,8,15,16,21,22-24}$

Las recomendaciones para el cierre primario incluyen puntos simples con monofilamentos, mantener la herida limpia y seca por 48 horas, y vigilancia cada dos días. ${ }^{15,24}$

\section{Antibióticos}

La posibilidad de infección depende de la naturaleza y sitio de la herida, así como de las características del individuo. La incidencia global de infección asociada con mordedura es de 10 a 20\%.4,6,7 Se observan mayores índices de infección en las siguientes circunstancias: mordeduras por gatos, cuando existe destrucción marcada del tejido, en heridas en sitios con pobre perfusión, lesiones en manos, pies y en aquéllas que involucren articulaciones, tendones, huesos, vasos o nervios. ${ }^{6,7,17}$ 
Existe una relación entre el tipo de herida y la incidencia de infección. En las heridas punzantes, se alcanza una incidencia de $31 \%$ en comparación con 6 y 5\% asociado con laceraciones y avulsiones, respectivamente. ${ }^{20}$

La profilaxis y terapia antibiótica es controversial. Se ha indicado en mordeduras de gatos, heridas punzantes profundas, lesiones en las que se haya realizado cierre primario, mordeduras en cara, manos y área genital, en lesiones próximas a articulaciones, en aquéllas con manejo tardío (12-24 horas en cara y 6-12 horas en brazos o piernas) y en pacientes inmunocomprometidos. $3,7,8$

Varios estudios han demostrado que la profilaxis no reduce significativamente las infecciones, excepto en las heridas que involucran a la mano, cuyo manejo profiláctico suele reducir la incidencia de 28 a 2\%.4,20

El esquema antibiótico que se ha utilizado como primera línea es amoxicilina/clavulanato en las primeras ocho a 12 horas durante tres a siete días, ${ }_{1}^{1,3,4,6,8,25}$ pues provee una adecuada cobertura para bacterias aeróbicas como estreptococos, S. aureus MS, Pasteurella spp. y E. corrodens; asimismo, también se ha recomendado contra bacterias anaeróbicas como Prevotella spp. y Porphyromonas spp (Tabla 3). ${ }^{9}$

Las alternativas en pacientes alérgicos a penicilinas son clindamicina más trimetoprima-sulfametoxazol (TMP-SMX) o cefalosporinas de amplio espectro en conjunto con clindamicina, o meropenem en monoterapia. ${ }^{6,9}$ En pacientes embarazadas que son alérgicas a la penicilina, se debe de considerar un esquema con TMP/SMX antes del tercer trimestre. ${ }^{23}$

En pacientes que muestren signos de infección sistémica (fiebre y escalofríos, celulitis progresiva y celulitis cerca de alguna articulación) se puede necesitar hospitalización y esquemas con antibióticos intravenosos, usando el esquema antibiótico de acuerdo con los patógenos causales más comunes, por ejemplo, en celulitis, los estafilococos y los estreptococos, teniendo como casos agregados algunas bacterias anaerobias; en caso de osteomielitis, se debe enfocarse el manejo a SARM, Pasteurella spp. y especies anaerobias. ${ }^{3,8,26}$ Los esquemas se prolongarán a dos semanas en casos que presenten celulitis; de dos a cuatro semanas, en caso de tendosinovitis, y de tres a seis semanas, en osteomielitis. 3,7

Infecciones por C. canimorsus y P. multocida responden adecuadamente a penicilinas y combinaciones de betalactámicos/inhibidores de betalactamasa. , $, 7,8$

\section{Vacunas \\ Vacuna del tétanos}

Es necesario administrar inmunoglobulina tetánica y toxoide tetánico a todos los pacientes que hayan sido mordidos y tengan al menos tres inmunizaciones. Se administrará obligadamente toxoide tetánico a pacientes con esquema completo que no hayan recibido una dosis entre cinco y 10 años; en caso de no haber recibido ninguna dosis en más de 10 años, la vacuna deberá aplicarse nuevamente. ${ }^{3,7}$

Vacuna de rabia

Las mordeduras de perros representan $97 \%$ de los casos de rabia en humanos. ${ }^{9}$ En Estados Unidos, sólo 45\% de

Tabla 3: Esquemas para manejo antibiótico.

Antibiótico de elección

Amoxicilina/ácido clavulánico

Clindamicina + trimetoprima/sulfametoxazol o cefalosporina tercer generación + clindamicina o meropenem en monoterapia

Trimetoprima/sulfametoxazol

Penicilina + clindamicina o vancomicina (SARM) + piperacilina/tazobactam

Cefalosporina tercera generación + metronidazol o clindamicina, meropenem o moxifloxacino en monoterapia

Vancomicina + cefalosporina de cuarta generación o meropenem + vancomicina
Espectro antimicrobiano

S. aureus MS, Pasteurella spp., E. corrodens, Prevotella y Porphyromonas spp.

Staphylococcus spp. MS, Streptococcus spp., anaerobios, Pasteurella spp.

Aerobios, poca cobertura contra anaerobios

Staphylococcus spp., Streptococcus spp., anaerobios

SARM, Streptococcus spp., Pasteurella spp. $\mathrm{y}$ anaerobios

SARM, Pasteurella spp. y otras bacterias anaerobias
Situación clínica

No alérgicos a penicilinas

Alérgicos a penicilina

Paciente embarazada antes del tercer trimestre

Celulitis

Tendosinovitis

Osteomielitis

MS = meticilino-sensible, SARM $=$ S. aureus resistente a meticilina. ${ }^{20,24}$ 
los perros y $8 \%$ de los gatos que causan las mordeduras requieren el esquema de inmunización; ${ }^{4}$ sin embargo, en México gracias a la Semana de Vacunación Nacional se ha observado un descenso significativo de rabia hasta de $81.6 \%$ de los casos por año, de los cuales en $33.7 \%$ de los pacientes se inicia tratamiento. ${ }^{26}$

Se recomienda la aplicación de esta vacuna a todas las personas que hayan sido expuestas a un animal con rabia. Ésta no está contraindicada en ninguna etapa del embarazo. La profilaxis incluye inmunoglobulina humana el día cero de exposición y vacuna en los días cero, tres, siete y 14 ; en México, se aplica una quinta dosis en el día 28 o 30, 3,4,7,17

La dosificación de gammaglobulina antirrábica humana se calcula de acuerdo con el peso del paciente: $20 \mathrm{UI}$ por kilogramo. ${ }^{27} \mathrm{Si}$ los pacientes han recibido previamente la vacuna, la inmunoglobulina no deberá administrarse y únicamente se administrará una vacuna en los días cero y tres. . $^{3,7,7,26}$

No es necesaria la vacuna si el animal no muestra signos o síntomas de rabia. Éstos incluyen: anorexia, disfagia, comportamiento anormal, parálisis o convulsiones; como precaución, deberá ser monitorizado al menos 10 días. ${ }^{4}$

\section{Ingreso}

Los criterios de ingreso hospitalario para tratamiento antibiótico endovenoso y tratamiento quirúrgico incluyen signos de sepsis, celulitis de instauración precoz (menor a 23 horas) linfangitis, linfadenitis, tenosinovitis, artritis séptica y osteomielitis. También se incluirán aquellos pacientes que tengan heridas que invaden la dermis, heridas punzantes profundas, heridas con repercusión funcional y cosmética y heridas que no mejoran tras tratamiento antibiótico ambulatorio.

\section{Seguimiento}

Todos los niños con heridas por mordedura necesitan ser reevaluados dentro de las primeras 24 a 48 horas posteriores para monitorear signos y síntomas de infección. ${ }^{1}$

\section{CONCLUSIONES}

Las mordeduras por animales continúan teniendo una alta causa de morbilidad en pacientes de todas las edades, presentándose como una de las 10 primeras causas de lesiones no fatales en niños de cinco a nueve años. ${ }^{27}$

El tratamiento de la mordedura por perro consiste en el manejo de la herida y tratamiento antibiótico. ${ }^{7}$ No obstante, actualmente, el tratamiento de cierre primario para las mordeduras es controversial. Antes, se mantenía la herida abierta para prevenir una infección por la sutura, sin embargo, se han hecho diferentes estudios en los que se debate el manejo primario, cursando con dos sugerencias clínicas: 1) cierre oportuno de herida y 2) mantener lesión abiertas o retrasar su cierre.

La discusión continúa abierta. Algunos estudios recomiendan el retraso del cierre primario en dos circunstancias: 1) cuando las mordeduras cursen con heridas punzantes en brazos y piernas y 2) cuando el manejo se ha retrasado más de 12 horas. Otra bibliografía recomienda el cierre de heridas para disminuir el riesgo de infección y mejorar los resultados estéticos; algunos más no encuentran diferencia en el riesgo de infección entre mantenerla cerrada o abierta, asegurando que esto dependerá del manejo adecuado y oportuno de la lesión.

La decisión del cierre y el uso de antibióticos deben de tomarse con base en el animal agresor, el tiempo en que se ha presentado quien haya sido mordido para la atención médica, las comorbilidades del paciente, el sitio y tipo de la lesión, así como los hallazgos clínicos de probables complicaciones.

\section{REFERENCIAS}

1. Álvez F. Infecciones por mordeduras y heridas punzantes. Protocolos diagnóstico-terapéuticos de la AEP: Infectología pediátrica. 3a. ed. Madrid, España: Asociación Española de Pediatría; 2011, pp. 177-188.

2. Hernández D. Mordedura de perro: enfoque epidemiológico de las lesiones causadas por mordedura de perro. Rev Enfer Infec Pediatr. 2009; 89: 13-20.

3. Rasmussen D, Landon A, Powell J, Brown GR. Evaluating and treating mammalian bites. JAAPA. 2017; 30 (3): 32-36.

4. Ellis R, Ellis C. Dog and cat bites. Am Fam Physician. 2014; 90 (4): 239-243.

5. Martin TCS, Abdelmalek J, Yee B, Lavergne S, Ritter M. Pasteurella multocida line infection: a case report and review of literature. BMC Infect Dis. 2018; 18 (1): 420.

6. Esposito S, Picciolli I, Semino M, Principi N. Dog and cat biteassociated infections in children. Eur J Clin Microbiol Infect Dis. 2013; 32 (8): 971-976.

7. Rothe K, Tsokos M, Handrick W. Animal and human bite wounds. Dtsch Arztebl Int. 2015; 112 (25): 433-443.

8. Aziz H, Rhee P, Pandit V, Tang A, Gries L, Joseph B. The current concepts in management of animal (dog, cat, snake, scorpion) and human bite wounds. J Trauma Acute Care Surg. 2015; 78 (3): 641-648.

9. Bula-Rudas FJ, OlcottJL. Human and animal bites. Pediatr Rev. 2018; 39 (10): 490-500.

10. Krau, SD. Bites and stings: epidemiology and treatment. Crit Care Nurs Clin North Am. 2013; 25 (2): 143-150.

Abrahamian, FM, Goldstein EJ. Microbiology of animal bite wound infections. Clin Microbiol Rev. 2011; 24 (2): 231-246.

12. Rueff F, Bedacht R, Schury G. Lesión por mordedura; clínica, tratamiento y proceso de curación. Med Welt. 1967; 12: 663-668.

13. Talan DA, Citron DM, Abrahamian FM, Moran GJ, Goldstein EJ. Bacteriologic analysis of infected dog and cat bites. Emergency medicine animal bite infection study group. N Engl J Med. 1999; 340 (2): 85-92.

14. Piccart F, Dormaar J, Coropciuc R, Schoenaers J, Bila M, Politis C. Dog bite injuries in the head and neck region: A 20-year review. Craniomaxillofac Trauma Reconstr. 2019; 12 (3): 199-204. 
15. Paschos NK, Makris EA, Gantsos A, Georgoulis AD. Primary closure versus non-closure of dog bite wounds. A randomised controlled trial. Injury. 2014; 45 (1): 237-240.

16. Singer AJ, Hollander JE, Subramanian S, Malhotra AK, Villez PA. Pressure dynamics of various irrigation techniques commonly used in the emergency department. Ann Emerg Med. 1994; 24 (1): 36-40.

17. Dendle C, Looke D. Animal bites: an update for management with a focus on infections. Emerg Med Australas. 2008; 20 (6): 458-467.

18. Thomson HG, Svitek V. Small animal bites: the role of primary closure. J Trauma. 1973; 13 (1): 20-23.

19. Hollander J, Valentine S, McCuskey C, Turque T, Singer A. Long-term evaluation of cosmetic appearance of repaired lacerations: validation of telephone assessment. Ann Emerg Med. 1998; 31 (1): 92-98.

20. Medeiros I, Saconato H. Antibiotic prophylaxis for mammalian bites. Cochrane Database Syst Rev. 2001; (2): CD001738.

21. Cheng HT, Hsu YC, Wu Cl. Does primary closure for dog bite wounds increase the incidence of wound infection? A meta-analysis of randomized controlled trials. J Plast Reconstr Aesthet Surg. 2014; 67 (10): 1448-1450.
22. Morgan M, Palmer J. Dog bites. BMJ. 2007; 334 (7590): 413-417.

23. Fleisher GR. The management of bite wounds. N Engl J Med. 1999; 340 (2): 138-140.

24. Stevens DL, Bisno Al, Chambers HF, Dellinger EP, Goldstein EJ, Gorbach SL et al. Practice guidelines for the diagnosis and management of skin and soft tissue infections: 2014 update by the Infectious diseases society of America. Clin Infect Dis. 2014; 59 (2): e10-52.

25. Giordano A, Dincman T, Clyburn BE, Steed LL, Rockey DC. Clinical features and outcomes of pasteurella multocida infection. Medicine (Baltimore). 2015; 94 (36): e1285.

26. Martínez M, Fernández J, Meléndez A, Vanda B, Edwards C, Chavira $M$ et al. Guía para la atención médica y antirrábica de la persona expuesta a la rabia. 2a. ed. México DF; 2010: pp. 1-42. Disponible en: https://www.gob.mx/salud/cenaprece

27. Shen J, Rouse, J, Godbole M, Wells HL, Boppana S, Schwebel DC. Systematic review: interventions to educate children about dog safety and prevent pediatric dog-bite injuries: a meta-analytic review. $J$ Pediatr Psychol. 2017; 42 (7): 779-791. 